\title{
The Day-After Effect: Long Term, Hebbian-Like Restructuring of Resting-State fMRI Patterns Induced by a Single Epoch of Cortical Activation
}

\author{
Tal Harmelech, ${ }^{1}$ Son Preminger, ${ }^{1,2 \star}$ Eliahu Wertman, ${ }^{3,4}$ and Rafael Malach ${ }^{1 \star}$ \\ ${ }^{1}$ Department of Neurobiology, Weizmann Institute of Science, Rehovot 76100, Israel, ${ }^{2}$ School of Psychology, Interdisciplinary Center 46150, Herzliya, Israel, \\ ${ }^{3}$ Department of Psychology, Hebrew University, Jerusalem 91905, Israel, and ${ }^{4}$ Department of Neurology, Hadassah Medical School, Hebrew University, \\ Jerusalem 91120, Israel
}

During rest, the cerebral cortex displays rich, coordinated patterns of spontaneous activity. The mechanism that shapes these patterns is largely unknown. Here we demonstrate that a Hebbian-like, sustained process plays a role in focusing these coherent patterns. Human subjects used an fMRI-based neurofeedback (NF) paradigm to intensely activate the dorsal anterior cingulate cortex for a single epoch $(30 \mathrm{~min})$. Resting-state correlations between all of the cortical voxels' BOLD time courses (functional connectivity) were mapped before, immediately after, and one day after the NF session. We found that the single epoch of cortical activation induced a lasting restructuring of the functional connections according to a Hebbian-like rule. Therefore, the change (increase and decrease) in functional connectivity strength of cortical voxels during rest reflected the level of their prior coactivation during the NF epoch. Interestingly, the effect was significantly enhanced $1 \mathrm{~d}$ after the NF activation epoch. The effect was evident in each subject individually, indicating its potential as a diagnostic window into the personal history of prior brain activations of both healthy and abnormal individuals.

\section{Introduction}

Even when subjects rest with eyes closed, the cerebral cortex displays a complex pattern of coordinated BOLD-fMRI "restingstate" activity. A remarkable feature of this activity is its organized nature (Greicius et al., 2003; Nir et al., 2006; Golland et al., 2008; Biswall, 2010; Mennes et al., 2010; Power et al., 2010; Shirer et al., 2011). Therefore, the correlation structures of spontaneous fluctuations follow the outlines of well known cortical networks (Greicius et al., 2003; Nir et al., 2006; Golland et al., 2008; Biswall, 2010; Power et al., 2010) and show a consistent interhemispheric connectivity (Nir et al., 2008). Despite a large body of research, the mechanism that underlies the spatial patterning of this spontaneously coordinated activity is still unknown. It has been demonstrated that cortical activation could restructure resting-state activity immediately after task performance (Albert et al., 2009; Lewis et al., 2009; Stevens et al., 2010; Tambini et al., 2010;

\footnotetext{
Received Dec. 30, 2012; revised April 11, 2013; accepted April 25, 2013.

Author contributions: T.H., S.P., and R.M. designed research; T.H. performed research; E.W. contributed unpublished reagents/analytic tools; T.H. analyzed data; T.H., S.P., and R.M. wrote the paper.

This work was funded by the Israel Science Foundation (Grant \#160/07 and Minerva grants to R.M. and Bikura Grant \#1538/09), the National Institute of Psychobiology in Israel (grant to S.P. and R.M.), the European Union (FP7 VERE grant), the Helen and Martin Kimmel Institute (award to R.M.), and the Weizmann Institute-Tel-Hashomer Medical Center (Sheba collaboration grant to S.P.). We thank Nahum Stern, Fanny Atar, and Dr. Edna Haran-Furman for their assistance in the imaging setup and rtfMRI data collection.

The authors declare no competing financial interests.

*S.P. and R.M. contributed equally to this work.

Correspondence should be addressed to: Rafael Malach, Department of Neurobiology, Weizmann Institute of Science, 234 Herzl Street, Rehovot 76100, Israel. E-mail: rafi.malach@weizmann.ac.il.

DOI:10.1523/JNEUROSCI.5911-12.2013

Copyright $\odot 2013$ the authors $\quad 0270-6474 / 13 / 339488-10 \$ 15.00 / 0$
}

Eryilmaz et al., 2011; Riedl et al., 2011; Zhang et al., 2012; Urner et al., 2013) or even at longer periods afterward (Taubert et al., 2011). However, the reported effects were mixed. For example, although Tambini et al. (2010) reported enhanced correlations after training in memory-related networks, Lewis et al. (2009) reported increased anticorrelations in visual to frontal cortex connectivity. We hypothesize that both increases and decreases in functional connectivity (FC) may be considered as two complementary aspects of a single cortical mechanism. We propose that the restructuring of resting-state connectivity follows a Hebbian-like learning rule. In Hebb's original formulation (Hebb, 1949), neuronal assemblies are generated through their successful coactivations. However, as both subsequent theoretical and experimental research has demonstrated in animal models, the complementary Hebbian modification is equally important and prevalent; that is, when activity between cortical neurons is decorrelated or even anticorrelated, the Hebbian-learning rule leads to a corresponding decrease in connectivity between the decorrelated neurons (Artola et al., 1990; Markram et al., 1997). Here we tested this hypothesis using an fMRI-based neurofeedback (NF) training method, which is known to produce strong activations resulting in significant brain-behavior changes even after a single session (Weiskopf et al., 2004; DeCharms et al., 2005; Johnston et al., 2010; Scharnowski et al., 2012). We trained subjects to enhance activity in the dorsal anterior cingulate cortex (dACC), an area implicated in volitional behavior (Rushworth et al., 2004; Holroyd and Coles, 2008). This protocol was chosen with the intent that a reorganization after such training may be 


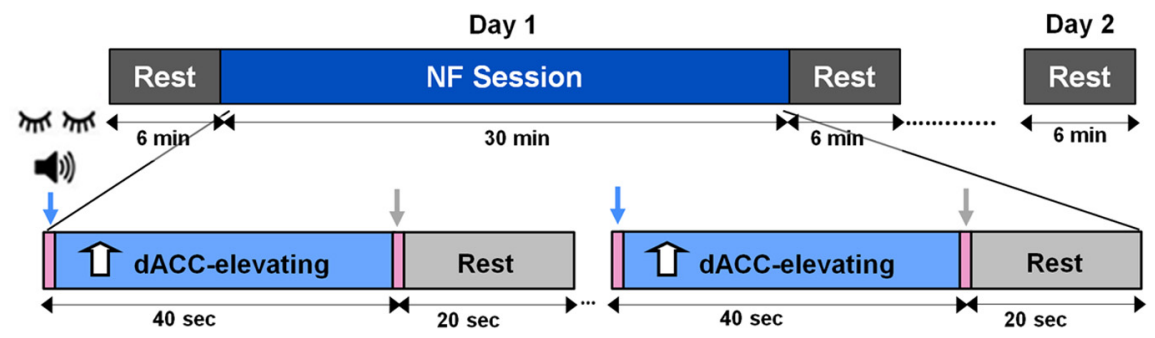

Figure 1. Experimental protocol. The experiment included two stages. On the first day, the subjects underwent an rt-fMRI NF session (30 min, dark blue epoch) and were scanned during rest before and after ( $6 \mathrm{~min}$, dark gray epochs). The NF session included three NF runs ( 10 min each); each run consisted of blocks of dACC-elevation conditions ( 40 s, light blue epochs) alternated with rest periods ( $20 \mathrm{~s}$, light gray epochs). During the NF sessions, auditory feedback was continuously provided via headphones. On the second day, the subjects were scanned during rest again (6 min, gray epoch). Subjects were scanned with closed and covered eyes and all epochs were indicated by brief ( $<1 \mathrm{~s}$ ) auditory cues (pink epochs, marked by arrows).

potentially valuable if applied to patients suffering from volitional impairments (Levy and Dubois, 2006). Rest scans preceded and followed the NF training, enabling us to determine the nature of resting-state $\mathrm{FC}$ restructuring.

\section{Materials and Methods}

Subjects. Twenty healthy subjects, all research students and research fellows (five females; mean age, $30.5 \pm 4.5$ years [range, 26-46]) participated in the experiment. All participants were right-handed, had normal hearing and normal or corrected-to-normal vision, and provided written informed consent for their participation. The Hertzog hospital ethics committee approved the experimental protocols.

Tasks and stimuli. The experiment was conducted in three separate days, with the last two days being consecutive (Fig. 1). On the first day, subjects were scanned while resting ( $6 \mathrm{~min}$ ) and subsequently underwent a real-time fMRI NF training session (30 min), after which they were again scanned during rest $(6 \mathrm{~min})$. On the following day, the subjects were scanned once more while at rest (6 min). Subjects' eyes were closed and covered during all scans.

Localizer. To localize the AACC region to be enhanced during training (see below), subjects performed two localizer tasks involving selfinitiation and volitional behavior, which were shown to activate this region (Rushworth et al., 2004; Holroyd and Coles, 2008; Mostofsky and Simmonds, 2008; Preminger et al., 2011).

A self-initiated action task (Preminger and Harmelech, 2008) consisted of intermittent blocks of voluntary action and blocks of determined action ( 60 s blocks of each condition) separated by rest periods ( 14 s). During the voluntary blocks, subjects viewed changing stimuli and, at the time of their choosing, could freely perform actions that have rewards and costs. The determined (control) game was similar to the voluntary game in actions, stimuli, and their timing, but the actions were determined by external stimuli. Voluntary and determined periods were presented in blocks of $60 \mathrm{~s}$, interleaved with each other, with $14 \mathrm{~s}$ blocks of black screen in between.

A self-initiated thought task (Preminger et al., 2011) consisted of seven blocks of volitional prospection in which subjects were asked to think about initiating a new project in their research (Preminger et al., 2011) intermittently with rest periods (40 and $20 \mathrm{~s}$, respectively) and blocks of reciting or recalling externally oriented content such as reciting the alphabet, the national anthem, or place imagery of a familiar place.

Neurofeedback training. On a subsequent day (average $2.6 \pm 0.54 \mathrm{~d}$ later), the subjects underwent an rtfMRI NF (Weiskopf et al., 2004; DeCharms et al., 2005; Johnston et al., 2010) session. The NF session consisted of 3 runs, with each run constituting a $7.5 \mathrm{~min}$ scan that included blocks of "dACC elevation" and rest (Fig. 1). For the dACC-elevating condition, subjects were given a general cognitive strategy ("volitional prospection," i.e., "think about initiating a new project in your research") that is known to activate this region (Preminger et al., 2011). Each block lasted $40 \mathrm{~s}$ and was followed by a $20 \mathrm{~s}$ rest period, both marked by brief $(<1 \mathrm{~s})$ auditory cues. During each run, subjects were provided with ongoing auditory feedback, a tone $2000 \mathrm{~ms}$ in length at every repe- tition time (TR) point, the pitch of which indicated the level of activity in the target region of interest (ROI) out of seven levels. For each TR, baseline was calculated by extracting the minimum and maximum values from a sliding window of the last 10 time points and subtracting them to calculate the dynamic activation range. The activation range was multiplied by 1.1 in case the activation level at a given time point exceeded that range. The relative level of activity was calculated as the ratio between the average ROI value for the given time point minus the minimum value, and the baseline as follows: given TR value $-\min / 1.1(\max -\min )$. Both the auditory cues and the real-time feedback were provided via headphones. Auditory feedback was chosen after a few pilot experiments with different types of feedback (two types of visual, auditory, and bimodal) were done. The feedback that yielded the best results (at least for this ROI) was the auditory feedback. The subjects also reported such auditory feedback to be less interfering with their mental strategy while being salient enough as feedback/for monitoring.

Rest scans. All the subjects were scanned during rest $(6 \mathrm{~min})$ at three time points of the experiment: before, immediately after, and $24 \mathrm{~h}$ after the NF session. The instruction to the subjects was as follows: "please rest. You can let your mind wander freely and think about whatever you'd like, except for the NF strategy." After each rest scan, the subjects were asked to give a description of their thought content during that scan.

Reports. After each resting and NF scan, the subjects were asked to freely describe their thought content/strategy during that scan. In addition, for the NF scans only, the subjects completed a questionnaire with four categories of questions regarding their experience during the dACCelevating condition. The categories included experiences of: control (e.g., "to what extent did you feel you were controlling your thoughts?"), self (e.g., "to what extent were your thoughts self-oriented"), success (e.g., "to what extent did you find yourself successful"), and modality (e.g., "to what extent were your thoughts visual"). A 5-point scale was used for this rating, for which $1=$ not at all and $5=$ a lot. Category-specific questionnaires were limited to the NF scan to minimize external induction of thought processes during the resting scans.

The subjects' free reports after the resting state scans could be divided (Buckner and Carroll, 2007) into two main content categories (1) "selfprojection," which included episodic memory and planning (examples of subjects' descriptions in this category are as follows: "I remembered a dinner with friends from the past weekend"; "I thought about what I need to get from the market"; and (2) "here and now," which included such things as "I was concentrating on the noise of the magnet" and "I was concentrating on sensory aspects of the blanket on my body and the headphones over my ears." A prominent category of such "here and now" thoughts consisted of repetitive inner speech such as "I was counting my breaths" or "I was repeating a mantra." These subjects will be referred to as the "repetitive speech" group in the following text. We used a blind scoring procedure, in which the reports from the three restingstate periods were intermixed. Ten naive raters who were unaware of which report belonged to which resting state independently rated the similarity between these reports and the reports of thoughts used during the NF period. The rating involved a 5 -grade scale for which $1=$ very similar and $5=$ very different. The instruction to the raters was simply to "rate the similarity of rest reports with NF reports on a scale of 1 to 5, 1 being very similar and 5 being very different." No strategy was given. Cronbach's $\alpha$ coefficient was calculated as a measure of internal consistency/interrater reliability of these content ratings.

Imaging setup. The scans were performed on a 3T Trio Magnetom Siemens scanner, at the Weizmann Institute of Science, Rehovot, Israel. The 3D T1-weighted anatomical images were acquired with highresolution $1 \mathrm{~mm}$ slice thickness (3D MP-RAGE sequence, $\mathrm{TR}=2300 \mathrm{~ms}$, $\mathrm{TE}=2.98 \mathrm{~ms}, 1 \times 1 \times 1 \mathrm{~mm}$ voxels $).$ BOLD contrast was obtained with gradient echo planar imaging sequence $(\mathrm{TR}=2000 \mathrm{~ms}, \mathrm{TE}=30 \mathrm{~ms}$, flip 
Table 1. Summary of all indices used in the analyses including their definitions, how they were calculated, and where they are used

\begin{tabular}{llll}
\hline Index name & Definition & Calculation & Where used \\
\hline Increment in FC & Change/difference in dACC resting-state FC & dACC FC in second rest - dACC FC in first rest & Figure 4, Figure 5 \\
Cl & Global correlation per voxel & Average correlation per voxel of all its TC correlations with all other voxel TCs & Figure 7A \\
Disparity index & Change/difference in Cl & Two-tailed paired $t$ test between Cl values in second and first rest & Figure 7B \\
\hline
\end{tabular}

angle $=90^{\circ}$, FOV $240 \mathrm{~mm}$, matrix size $80 \times 80$, scanned volume $=35$ axial slices of $4 \mathrm{~mm}$ thickness, no gap, $3 \times 3 \times 4 \mathrm{~mm}$ voxels, anterior commissure-posterior commissure).

Data analysis and preprocessing. RtfMRI data were analyzed with the "Turbo Brain-voyager" and "Brain-voyager" software packages (Brain Innovation) and with complementary inhouse software. The first two images of each functional scan were discarded to allow for T1-equilibration effects. The functional images were superimposed on 2D anatomic images and incorporated into the $3 \mathrm{D}$ datasets through trilinear interpolation. The cortical surface in a Talairach coordinate system (Talairach and Tournoux, 1988) was reconstructed for each subject from the 3D-spoiled gradient echo scan. Data were corrected for slice-dependent time shifts for each volume and 3D head motion. Motion parameters generated in the latter process were used later as nuisance regressors in the general linear model (GLM) and correlation analyses. fMRI images from the localizer tasks and the NF session were spatially smoothed with a Gaussian kernel with a full width at half maximum (FWHM) of $4 \mathrm{~mm}$. Temporal filtering of linear trends was performed to account for LF noise (e.g., scanner drift). All regressors were modeled as

box-car functions convolved with the hemodynamic response function. A hemodynamic lag of 3-6 s was assumed and verified for each subject. The analysis was performed independently for the time course of each individual voxel. After computing the coefficients for all regressors, a Student's $t$ test between coefficients of different conditions (e.g., dACC elevation vs rest) was performed. Multisubject analysis was based on a random-effect GLM (Friston et al., 1999). The multisubject functional maps were projected on an inflated or unfolded Talairachnormalized brain. Significance levels were calculated taking into account the minimum cluster size and the probability threshold of a false detection of any given cluster. This was accomplished by a Monte Carlo simulation (cluster-level statistical threshold estimator in "Brain-voyager") using the combination of individual voxel probability thresholding. The probability of a false-positive detection per image was determined from the frequency count of cluster sizes within the entire cortical surface (NF session had a threshold of 184 voxels, the self-initiated thought localizer task had a threshold of 183 voxels, and the self-initiated action localizer task had a threshold of 118 voxels; $\alpha$ was set at 0.05 throughout). Spatial smoothing with a Gaussian kernel with an FWHM of $8 \mathrm{~mm}$ was used for all multisubject maps.

Definitions of ROIs. NF session target ROI (left dACC) was defined individually for each subject as the region of overlap $(r=15 \mathrm{~mm})$ between the self-initiated action and the self-initiated thought task activations. Neutral ROI (left middle frontal gyrus) was defined individually for each subject as a region $(r=15 \mathrm{~mm})$ neighboring the NF target ROI that was not activated during the NF. The second most activated ROI (left inferior parietal lobule [IPL]) was defined individually for each subject as a region $(r=15 \mathrm{~mm})$ that was the second most activated during the NF (the first being the target ROI in dACC). The group-level ROIs are shown for presentation purposes in the figures; however, for the actual calculations, measurements from individually defined subjects' ROIs were used for all the analyses.

Statistical analysis of behavioral measures. Correlations of subjects' reports with fMRI data were performed using nonparametric Spearman's rank order tests. FDR correction for multiple comparisons was done according to the Benjamini-Hochberg method (Benjamini and Hochberg, 1995). $\alpha$ was set at 0.05 .

FC maps. After preprocessing, the fMRI data from the rest runs were subjected to additional processing steps, as described previously (Fox et al., 2005; Vincent et al., 2006; Van Dijk et al., 2010), before FC analysis of low-frequency BOLD fluctuations. First, a temporal bandpass filter was applied to the atlas aligned BOLD data, retaining signal within the frequency range of $0.009-0.08 \mathrm{~Hz}$. Data were then spatially smoothed with a Gaussian kernel (FWHM $=4 \mathrm{~mm}$ ). Sources of variance of noninterest were then removed from the data by regression of nuisance variables (in addition to first temporal derivatives of each), including: the six motion parameters obtained during the motion correction procedure, the mean signal from the lateral ventricles, and the mean signal from a region within the deep cerebral white matter.

To perform the FC analysis, seed time series were sampled, averaged, and $z$-transformed for all voxels in the NF peak activation ROI (see ROI definition for details). The resulting time series were then used as predictor after removal of the auto-regression factor model in a single-subject GLM. A second-level random effect analysis was then performed to determine brain areas that showed significant FC with the NF peak ROI 
A

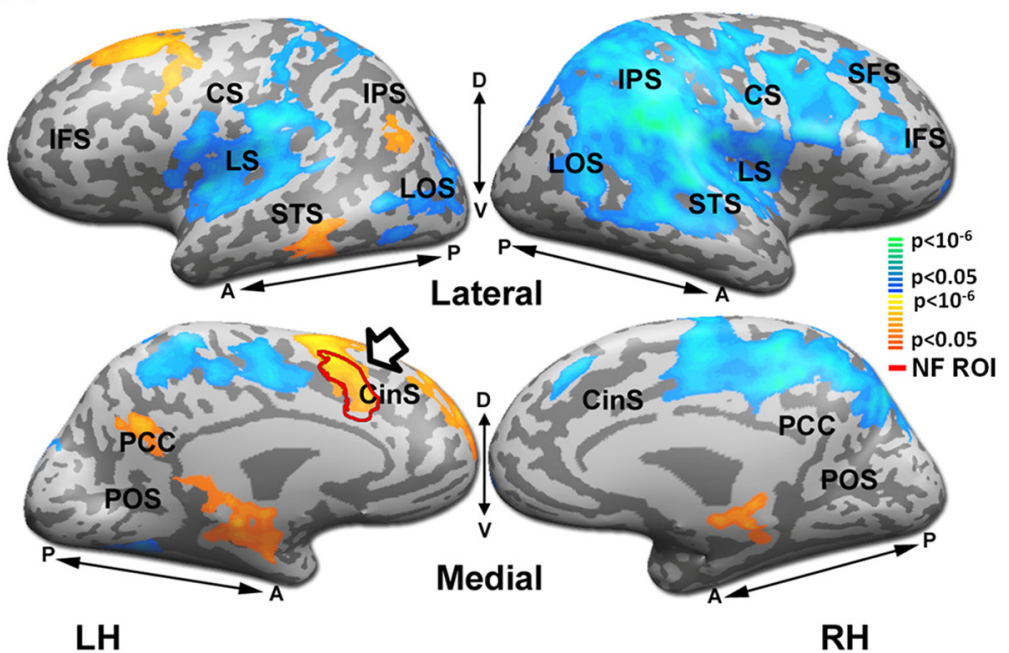

B

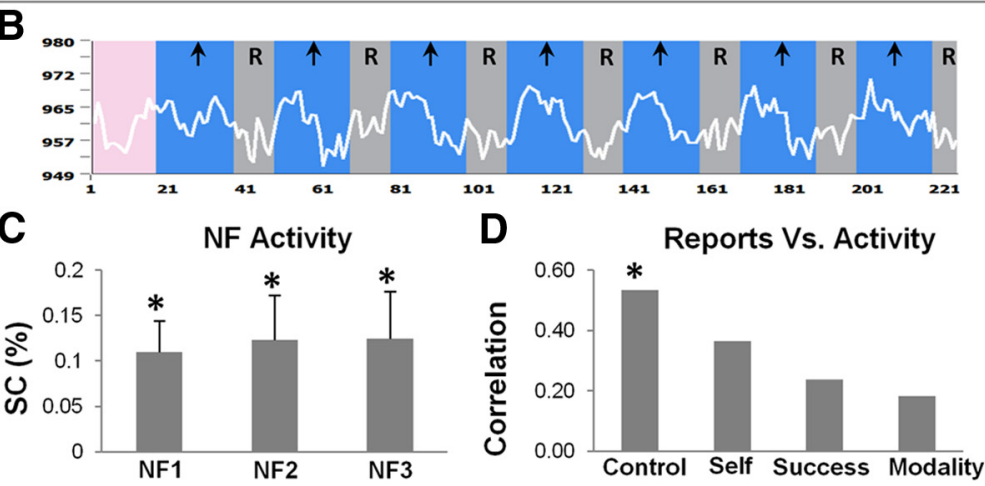

Figure 3. NF session. A, Activity maps for the NF session dACC-elevating versus rest. Activity maps from multisubject $(n=20)$, random-effect GLM analysis (corrected for multiple comparisons) are presented in lateral (L) and medial (M) inflated format. Color scale indicates statistical significance. Yellow-orange regions represent areas that were preferentially activated in the $\mathrm{dACC}$-elevating blocks, whereas blue-green regions represent areas that were preferentially activated during rest. The NF target ROI (dACC, defined for each subject individually, see Materials and Methods) is indicated by red contour and arrow. LH indicates left hemisphere; $\mathrm{RH}$, right hemisphere; $\mathrm{M}$, medial; $\mathrm{L}$, lateral; $\mathrm{A}$, anterior; $\mathrm{P}$, posterior; CS, central sulcus; SFS, superior frontal sulcus; CinS, cingulate sulcus; LTC, lateral temporal cortex; IFS, inferior frontal sulcus; IPS, intraparietal sulcus; LS, lateral sulcus; PCC, posterior cingulate cortex; P, posterior. $\boldsymbol{B}$, Example NF time course from the dACC for a typical subject. Blue and gray epochs represent $\mathrm{dACC}$-elevating blocks and rest periods, respectively. $C$, Average activity from dACC for $\mathrm{dACC}$-elevating condition for each NF run. Asterisks denote statistical significance relative to rest baseline. Error bars indicate SEM. ${ }^{*} p<0.05$. $D$, Correlation between subjects' $d A C C$ BOLD activation and their reports regarding their NF strategy (corrected for multiple comparisons). Asterisk denotes statistical significance. ${ }^{*} p<0.05$. For the questionnaire details, see Materials and Methods.

across all subjects during the experiment. To perform a two-tailed paired $t$ test to determine significant voxel-by-voxel correlation differences between the rest periods, the correlation coefficients were converted to $z$ values using Fisher's $r$-to- $z$ transformation to improve normality. The resulting $t$ values were projected onto a representative individual's cortical surface, forming FC disparity maps. A correction for multiple comparisons was performed by calculating the probability of a false positive from the frequency count of cluster sizes within the entire cortical surface using a Monte Carlo simulation as noted above (the presession rest had a threshold of 181 voxels, the postsession rest had a threshold of 185 voxels, and the day-after-session rest had a threshold of 176 voxels; $\alpha$ was set at 0.05 throughout). Spatial smoothing with a Gaussian kernel with an FWHM of $8 \mathrm{~mm}$ was used for all multisubject maps.

Hebbian-like sharpening analysis. To examine the quantitative relationship between the cortical activation during the NF and the changes in resting-state FC, a whole cortex analysis was conducted as follows. For each subject and each cortical voxel, the level of its coactivation with the target ROI (Fig. $5 B$ ) during the NF training was calculated. The relationship between this coactivation level and the increment in FC (Table 1) $1 \mathrm{~d}$ after the training was then plotted. The significance of each scatter plot was determined by a two-tailed Student's $t$ test. To avoid overestimation of the $p$-value due to the spatial smoothing of the data, the analysis was also performed on unsmoothed data. Before comparing the effect immediately after the NF session to $1 \mathrm{~d}$ after the NF session, the correlation coefficients were converted to $z$ values using Fisher's $r$-to- $z$ transformation to improve normality and then a two-tailed paired $t$ test was performed to determine significant differences between the immediate and latent effects. The same procedure was performed for the comparison between the effects of the target ROI (dACC) and the second most activated ROI (IPL).

Global FC: single-subject correlation index. For each subject's functional scan, the time course of each cortical voxel was transformed to units of percentage signal change, correlated with the time courses of all other cortical voxels, and then the correlations were averaged for each voxel. Voxels with a mean signal intensity level lower than 100 were discarded as noise, because regions outside the brain show similar values. This procedure (Fig. 7A, modified from Salomon et al., 2011) yielded a single correlation index (CI) per voxel (Table 1), which represented the degree of correlation between the voxel and all other cortical voxels. The CIs of all cortical voxels were then presented in cortical map form.

Global FC: group-level analysis and disparity index. For each voxel, the average CI was computed separately for each rest period by averaging the CIs of that voxel between all the subjects. Coregistration of the subjects' voxel space was done in the Talairach coordinate system (Talairach and Tournoux, 1988) on the 3D volume space. The resulting averaged voxel correlations were then projected onto a representative individual's cortical surface. Disparity indices (Table 1) were computed to measure the degree of difference between the rest periods in terms of the average CI of each voxel. The gray matter cortical voxels of all subjects were intersected and the correlation coefficients were converted to $z$ values using Fisher's $r$-to- $z$ transformation to improve normality. The CIs of all voxels were then extracted and a two-tailed paired $t$ test was performed to determine significant voxel-by-voxel correlation differences between the rest periods. The resulting $t$ values were projected onto a representative individual's cortical surface, forming FC disparity maps. These maps show voxels in which the presession rest period had significantly higher FC than the day-after-session rest period. A correction for multiple comparisons was performed by calculating the probability of a false positive from the frequency count of cluster sizes within the entire cortical surface using a Monte Carlo simulation as noted above (threshold of $180 \mathrm{~mm}^{2}, \alpha$ set at 0.05 ).

\section{Results}

\section{Brain activity during rtfMRI-NF session}

Brain activity during the tasks localizing the dACC is depicted in Figure 2. For each subject, the region that was chosen for the NF procedure was the overlapping activation in the two tasks in the dACC (arrow, overlap in red, for details, see Materials and Methods). 
A

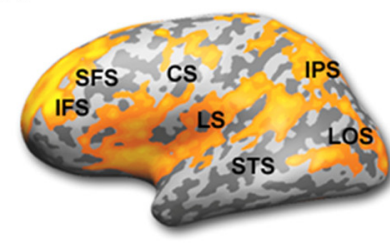

B

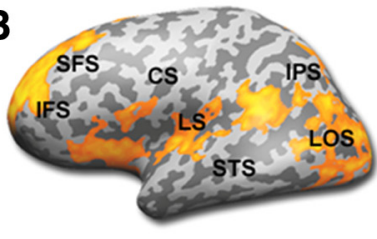

C

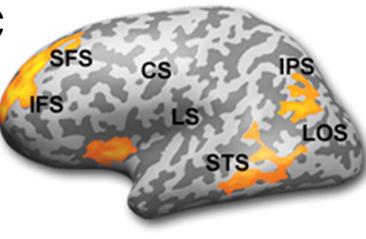

D

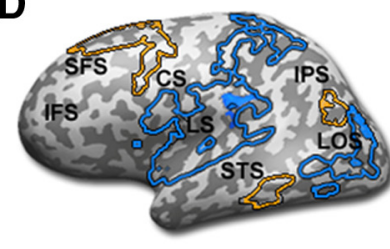

\section{Resting state functional connectivity}
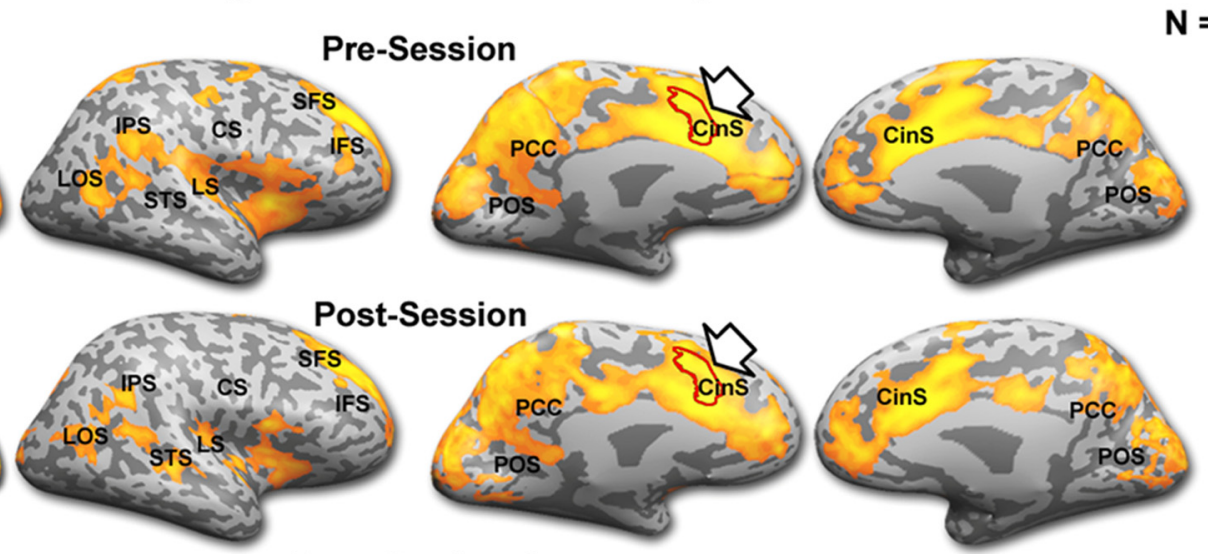

Day-after Session
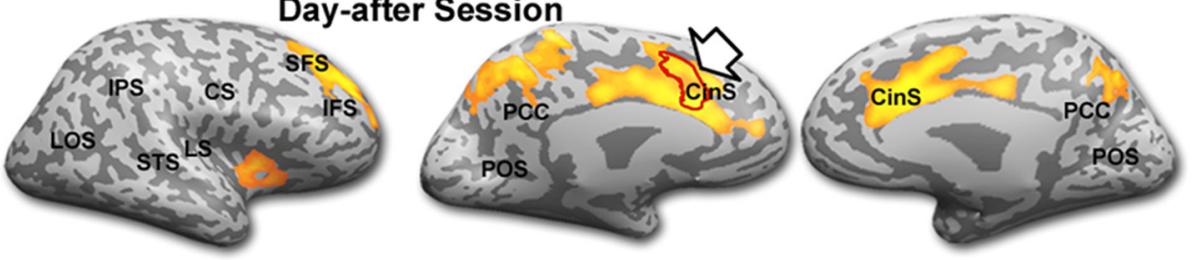

$\mathbf{N}=\mathbf{2 0}$
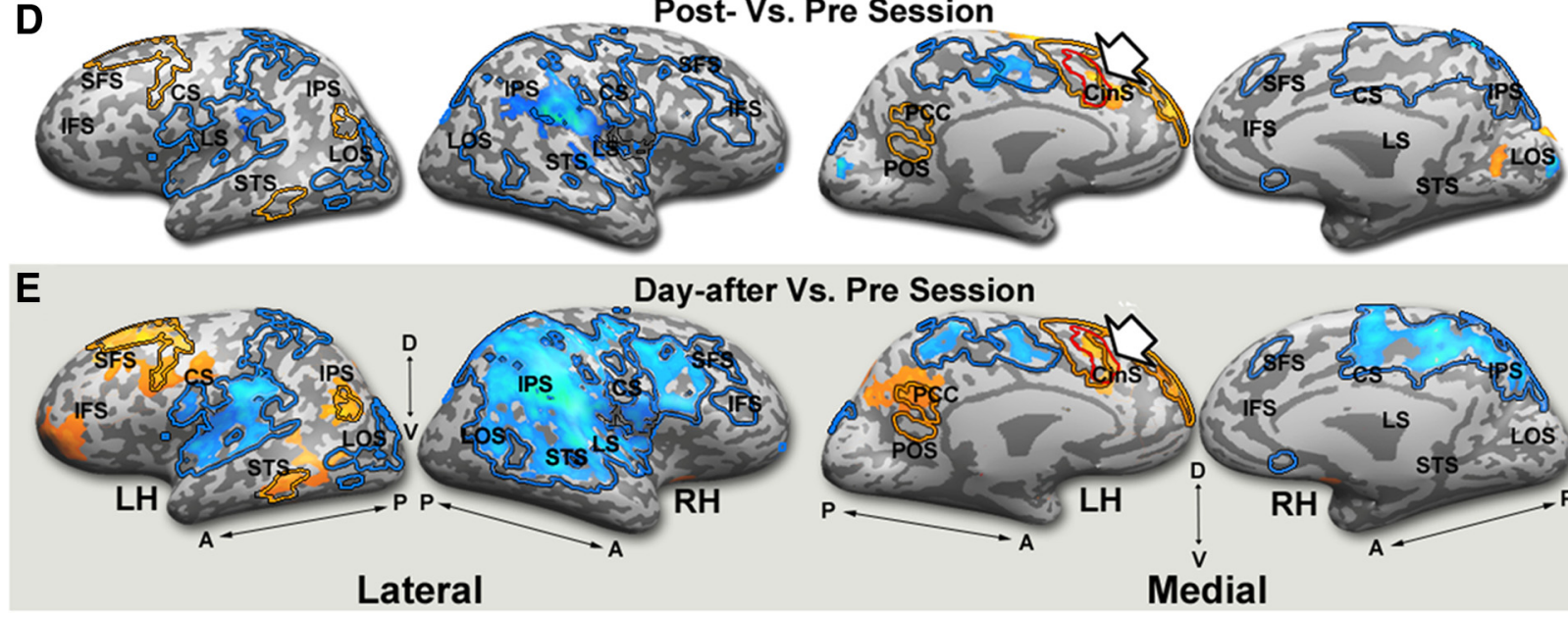

s. Pre Session

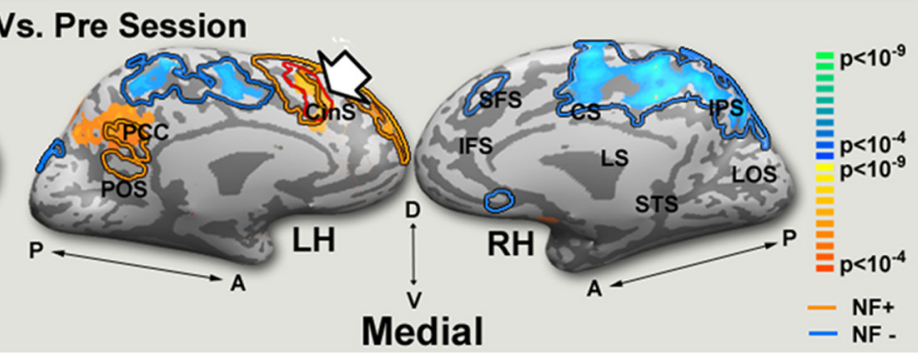

Figure 4. Resting-state FC changes after the NF session. Resting-state FC maps for the NF target ROI (defined per-subject, indicated by red contour and arrow), before $(\boldsymbol{A})$, immediately after ( $\boldsymbol{B})$, and $1 \mathrm{~d}$ after $(\boldsymbol{C})$ the NF session. All maps are multisubject $(n=20)$ and corrected for multiple comparisons. Comparing the maps from $\boldsymbol{B}$ and $\boldsymbol{C}$ showed that the effect was significantly stronger on the second day $(p<0.001)$. D, Statistical analysis (two-tailed paired $t$ test) of the difference in $\mathrm{FC}$ immediately after the NF session. $\boldsymbol{E}$, Statistical analysis (two-tailed paired $t$ test) of the difference in FC $1 \mathrm{~d}$ after the NF session. Color scale indicates significance. Red and blue contours represent activation and inactivation during the NF session, respectively. LH indicates left hemisphere; RH, right hemisphere; M, medial; L, lateral; A, anterior; P, posterior; CS, central sulcus; SFS, superior frontal sulcus; CinS, cingulate sulcus; LTC, lateral temporal cortex; IFS, inferior frontal sulcus; IPS, intraparietal sulcus; $L S$, lateral sulcus; $P C C$, posterior cingulate cortex.

Figure 3 depicts the results of the NF experiment (see Materials and Methods). Figure $3 B$ shows an example time course in one such session obtained from the target dACC ROI showing the successful BOLD activation during NF periods. The effect was robust and consistent across subjects, as depicted in the group map (Fig. 3A). High activation in the target ROI (arrow), as well as some additional regions in prefrontal, parietal, and temporal cortices (the IPL, superior frontal gyrus, middle frontal gyrus, and middle temporal gyrus) likely overlapping the default mode network, can be seen. Cross-scan analysis revealed a significant NF activation, with similar activation levels as the session progressed (Fig. 3C). Figure 3D depicts subjects' reports of experience during the NF (see questionnaire details in Materials and Methods), with experience of control over thought being significantly correlated with level of activation.

\section{Resting-state FC changes after rtfMRI-NF}

Our central focus was the possible changes in FC-resting-state correlations associated with the NF target ROI after the NF session. Figure $4 A-C$ depicts the FC of the NF target ROI before, immediately after $(\sim 2 \mathrm{~min})$, and $1 \mathrm{~d}$ after $(24 \mathrm{~h}$ later) the $\mathrm{NF}$ session. As is evident even by a qualitative inspection, the anatomical spread of FC was reduced after the NF session and this spread constricted further $1 \mathrm{~d}$ after the NF session. Figure $4 D, E$ show statistical analysis of the difference (two-tailed paired $t$ test) in FC before versus immediately after and before versus $1 \mathrm{~d}$ after the NF session, respectively. The contours on top of these disparity maps represent the activity during the NF session. The overlap displayed shows a decrease in FC in regions that were inactivated during the NF session as opposed to an increase in the activated 
A
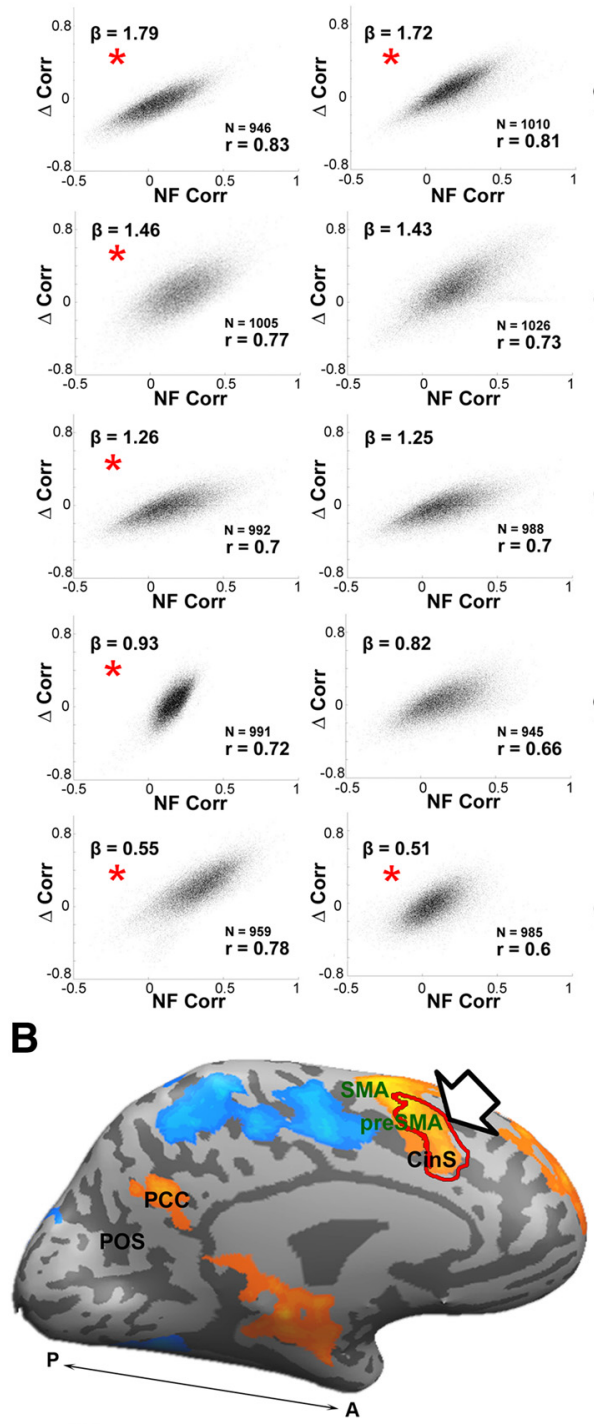

LH

\section{Individual subjects' "Gallery"- NF ROI}
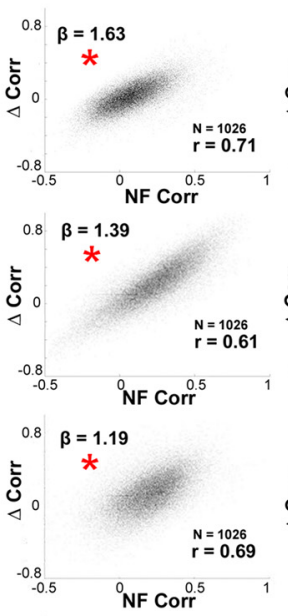

$0.8 \quad \beta=0.75$
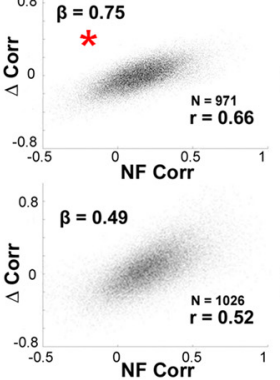

C

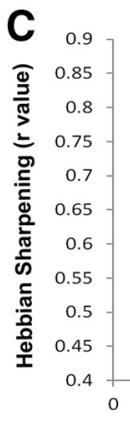

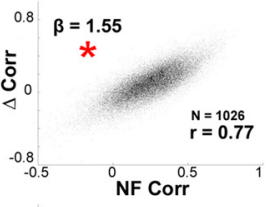

0.8. $\begin{gathered}\beta=1.38 \\ \star\end{gathered}$

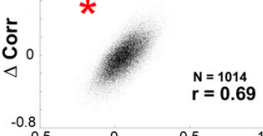

$\stackrel{-0.8}{-0.5} \quad{ }^{0} \mathrm{NF}$ Corr

$0.8 \beta=1.03$

*

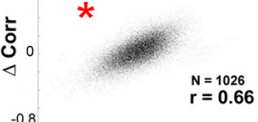

$\begin{array}{ll}-0.8 & 0.5 \quad \text { NF Corr } \\ 0.5 & 1\end{array}$

$0.8 \quad \beta=0.75$
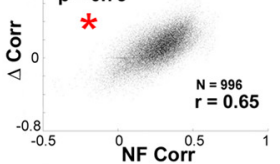

$0.8 \quad \beta=0.35$

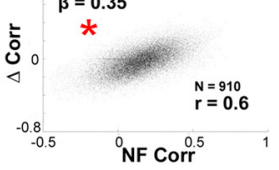

${ }^{*} p<5^{*} 10^{-4}$ for all subjects
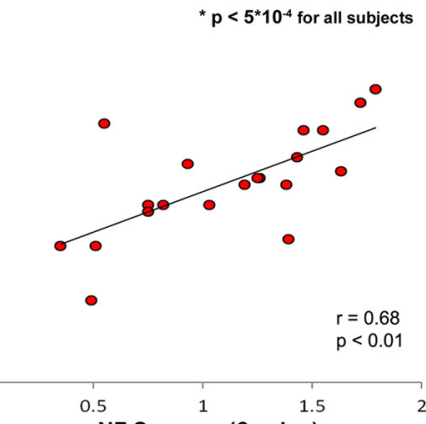

NF Success ( $\beta$ value)

Figure 5. Hebbian-like focusing of resting-state correlations (functional connectivity) induced by a localized cortical activation. $A, A$ "Gallery" of all 20 subjects who participated in the study. Each panel plots, for all cortical voxels (total $n=19,897$ voxels), the relationship between a voxel's coactivation with the target ROI (dACC) during NF training ( $x$-axis, measured as linear correlation) versus the change in its resting-state connectivity to the dACC a day after the training ( $y$-axis, correlation increment). Note that in each and every subject, we find a highly significant $(p<0.005)$ correlation between training activation and change in connectivity. Thus, voxels that were coactivated during training increased their resting-state connectivity a day after training, while those that were decorrelated during training decreased it. Red asterisks indicate subjects whose reported thought content during rest was repetitive inner speech. $\boldsymbol{B}$, The NF target ROl, shown by arrow and represented by a red contour. $\boldsymbol{C}$, A scatter plot demonstrating the relationship between the individual subjects' success in NF activation and their correlations from $A$. $\beta$ indicates the level of target NF activation during training.

regions. To examine the quantitative relationship between the cortical activation during the NF and the changes in resting-state FC, we conducted a whole cortex analysis (see Materials and Methods). For each subject and each cortical voxel, we calculated the level of its coactivation with the target ROI (Fig. 5B) during the NF training. We then plotted the relationship between this coactivation level and the increment in FC (Table 1) $1 \mathrm{~d}$ after the training. We found a highly significant correlation that was consistently evident in every subject who participated in the study. To represent this consistency across individuals, the scatter-plots are presented as a "gallery" of individual subjects (Fig. $5 A$ ). It is evident that in every subject who underwent training, there was a highly

significant $(p<0.005)$ relationship between the level of coactivation during the NF training and connectivity increment (or decrement) in the resting-state connectivity. To avoid overestimation of the $p$-value due to the spatial smoothing of the data, the analysis was also performed on unsmoothed data. The $p$-values reported are the more conservative estimates. Furthermore, the results remained significant $(p<0.05)$ even after reducing the number of degrees of freedom by a conservative fivefold.

Comparing these correlations for the rest period immediately after the training (mean $0.49 \pm 0.02, p<0.01$ ) and the one $1 \mathrm{~d}$ after the training showed that the effect was significantly stronger on the second day $(p<0.001$, with only 1 subject of 20 showing the opposite trend).

\section{Relationship of FC change to NF activation}

We also examined whether the relationship between NF coactivation and resting-state FC modulation was dependent on individual subjects' success in activating the NF target ROI during the training. The results are depicted in Figure $5 C$, in which the level of FC "Hebbian sharpening" (reflected in the individual correlation coefficients of the panels in Fig. 5A) in each of the subjects is plotted against their NF success (measured as the GLM $\beta$ values). Statistical analysis showed that the relationship between the two phenomena was highly significant $(r=$ $0.68, p<0.01$ )

Was the Hebbian sharpening localized to the NF target site or was it present also in neighboring cortical regions, depending on their level of activation by the NF task? To answer this, we conducted two control analyses with two different ROIs as the targets for the analyses. The first ROI we chose was a neighboring neutral (nonactivated, see Materials and Methods) ROI as the target for the analysis. Repeating the correlation analysis in identical fashion to that conducted for the dACC ROI (Fig. 5) failed to show any significant sharpening effect in the correlation to the "sham" cortical site. The results are depicted in Figure 6. Comparing Figure 5 and Figure 6 again illustrates the consistent, subject-by-subject difference between the target and control sites. To examine the possibility of a rapidly decaying focusing effect in the sham cortical site, we repeated the analysis for the rest immediately after the NF period. Again, no significant focusing effect was discerned (mean $0.08 \pm 0.033, p=$ 0.1 , and $0.09 \pm 0.036, p=0.09$, for the postsession and day-after, respectively, and $p=0.45$ for the difference).

In addition, we analyzed activity in another ROI, the region that was the second most activated region during the NF (see Materials and Methods). We found the same effect, albeit to a lesser extent (mean $0.42 \pm 0.04, p<0.01$, and $0.51 \pm 0.02, p<0.005$, for the 
postsession and day-after, respectively, and $p<0.01$ for the difference).

\section{Selectivity of the Hebbian-like sharpening effect}

To further examine whether the Hebbian sharpening was selective to the NF activation site or reflected a more global cortical phenomena, we mapped changes in global FC for each cortical voxel. We analyzed the three rest periods for each subject individually as follows (Fig. 7A). For every cortical voxel in each subject, we assigned a CI by computing the correlation of that voxel with all other cortical voxels. This resulted in a CI map, in which voxels were assigned high or low values depending on how widespread their correlations with the rest of the cortex were. We then averaged these CI maps across individuals for each of the rest periods to generate a multisubject CI map for each period (see Materials and Methods). These CI maps were then compared across the rest periods to reveal changes in global connectivity. This comparison enabled us to identify, in a data-driven fashion, those cortical regions that underwent a significant FC change after the NF activation (Fig. 7A, see Materials and Methods). The results of this analysis are depicted in Figure $7 \mathrm{~B}$. As can be seen, the region that underwent the most prominent change in global connectivity after the training largely overlapped the NF activation site.

\section{Assessment of subjects' cognitive state during rest}

Was the subjects' cognitive state during the resting-state measurements affected by the prior NF training? To examine this issue, we used four complementary approaches. First, subjects were interviewed about their thought content after each rest period (see Materials and Methods for details). Ten naive raters who were unaware of which report belonged to which resting state rated the similarity between these reports and those obtained for the NF period. The rating involved a 5-grade scale for which $1=$ very similar and $5=$ very different. The reports for all three rest periods were rated as very different from the NF strategy (average presession, $4.81 \pm 0.08$; immediate postsession, $4.85 \pm 0.06$; day-after-session, $4.91 \pm 0.04$, respectively). The internal consistency of the content ratings was very high (Cronbach's $\alpha=0.92$ ). Furthermore, no correlation was found between the success in NF activation and the resting-state thought content $(r=0.07, p=0.8)$.

Second, we divided the rest reports into two categories, which appeared to differ in their similarity to the NF strategy (see Materials and Methods). Comparing the sharpening effects for reports from the two categories failed to show any significant difference (mean similar, $0.64 \pm 0.035$; different, $0.69 \pm 0.03$, $p=0.79)$. Furthermore, no correlation was found between the
Individual subjects' "Gallery"- Control ROI
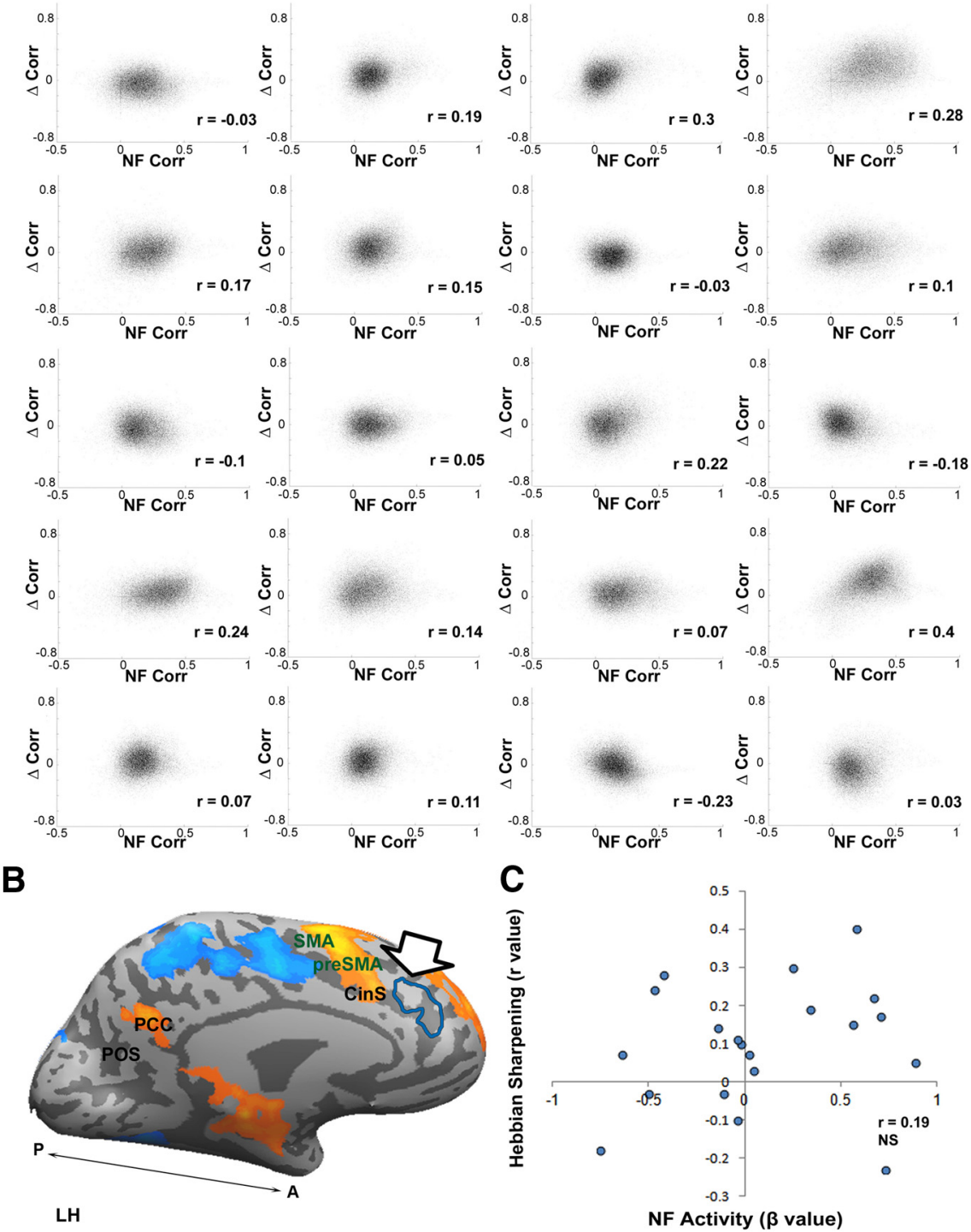

Figure 6. Lack of Hebbian-like focusing in a control ROI. $\boldsymbol{A}$, "Gallery" of all 20 subjects who participated in the study. The analysis was identical to the one conducted for Figure $4 A$, except that here the target region was not the NF-activated area (dACC), but a control region (left middle frontal gyrus) neighboring the $\mathrm{dACC}$ that was not activated during the NF. Note the lack of sharpening contour. C, Scatter plot demonstrating the lack of relationship between the individual subjects' level of activation in the control ROI during the NF session and their correlations from $\boldsymbol{A}$. $\beta$ indicates the level of control NF activation during training.

rest and NF reports' similarity rating and the sharpening effects $(r=0.08, p=0.74)$.

Third, we identified a specific group of subjects termed "repetitive speech," whose thought content appeared clearly remote from the NF strategy (see Materials and Methods). The Hebbian focusing effect for this selected group is depicted in red frames in Figure $5 \mathrm{~A}$. As can be seen, no bias for lower sharpening level in this group of subjects can be discerned.

Fourth, comparing the amplitude of BOLD fluctuations during the NF session with those obtained during the rest periods revealed that the fluctuations were significantly higher than both the presession and day after rest periods $(p=0.001$ and $p=0.02$, paired $t$ test, respectively, Bonferonni corrected). We took advantage of this observation to examine, in an objective manner, whether there was a "replay" of the NF task during the subsequent rest periods. Compar- 


\section{A Subject level analysis}

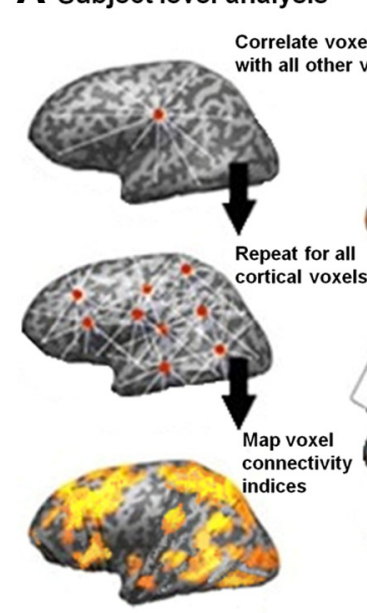

xel TC

\section{Group level analysis}

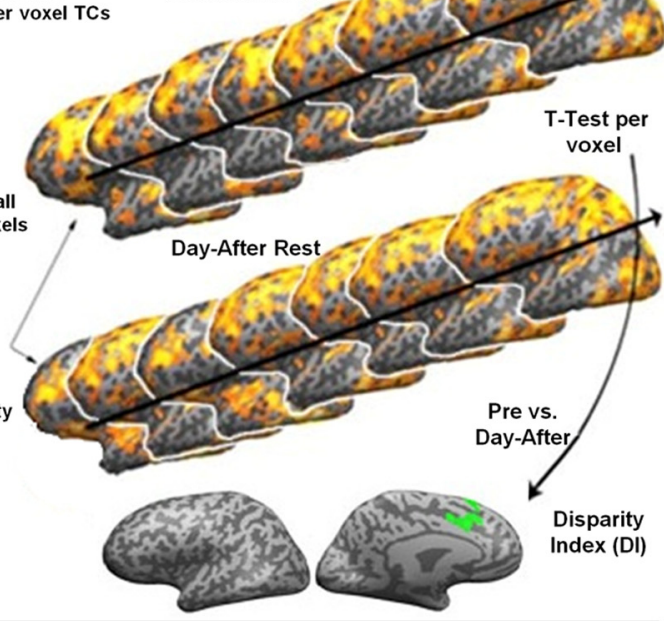

$\bar{B}$

\section{B (1)}

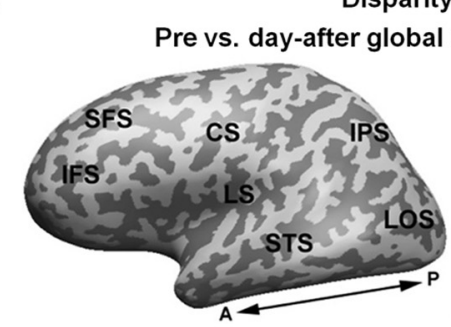

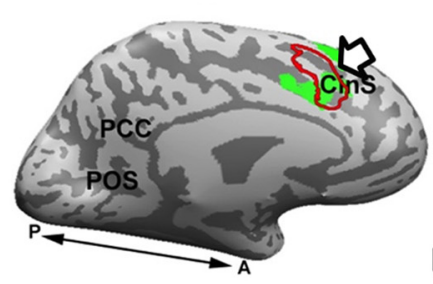

LH
$\mathrm{N}=20$

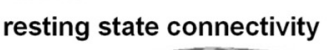

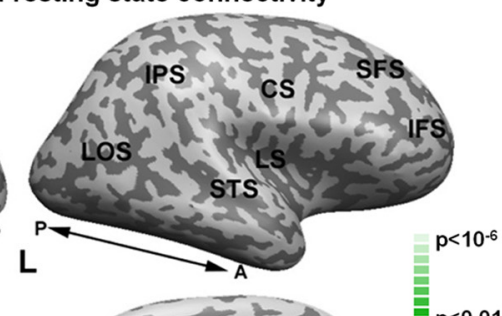

M

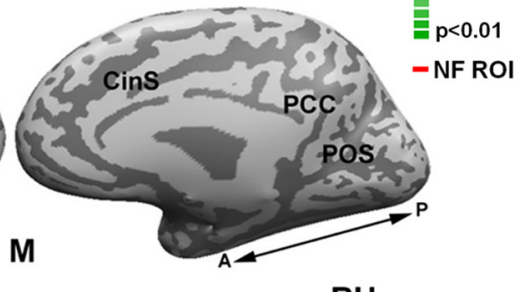

RH

Figure 7. Selectivity of FC-focusing effect. $\boldsymbol{A}$, Scheme of data analysis pipeline (see Materials and Methods). Left, Subject-level analysis. For each subject's functional scan, each voxel's time course was correlated with the time course of all other voxels (top). This was repeated for all cortical gray matter voxels, so that each voxel had a single Cl that was the mean of its correlation with all other voxels (Cl, middle). The voxel Cl was projected onto spatial maps (bottom). Right, Group-level analysis. The voxel connectivity maps for all subjects in each group were subjected to a voxel-by-voxel paired $t$ test. The resulting $\mathrm{FC}$ disparity maps show voxels in which one group had significantly higher FC. $\boldsymbol{B}$, Disparity map of the presession versus day-after-session global resting-state FC (green). NF peak ROIs are indicated by red contour and arrow. Color scale indicates statistical significance.

ing the BOLD fluctuations during the rest periods before and $1 \mathrm{~d}$ after the NF training failed to reveal a significant difference ( $p=$ 0.55 , paired $t$ test). These results argue against the possibility that the observed FC changes were due to subjects' "replaying" the $\mathrm{NF}$ task during the rest period $1 \mathrm{~d}$ after the NF training.

\section{Discussion}

Our study demonstrates that the pattern of resting-state activity can be reorganized after a single intense epoch of cortical activation. The reorganization process followed a remarkably consistent, unitary, "Hebbian" rule. Therefore, voxels that were coactivated during training increased their resting-state connectivity $1 \mathrm{~d}$ after training, whereas those that were decorrelated during training, decreased it. Furthermore, the effect appeared to be linearly related to the magnitude of the co-modulation (Fig. 5). Therefore, the main characteristic of the FC restructuring can be described as Hebbian-like sharpening. The overall consequence of intense cortical activation was thus to "focus" the patterns of resting-state correlations (FCs) around the most activated sites. This conclusion was further supported by the fact that, in our purely data-driven analysis of connectivity, the main site showing reduced global connectivity overlapped the NFactivated region (Fig. $7 B$ ).

Our results provide a unifying framework to the substantial body of literature describing such restructurings, in which some FC changes were reported to be of deeper anticorrelation (Lewis et al., 2009), whereas others reported increased FC levels (Albert et al., 2009; Tambini et al., 2010; Taubert et al., 2011) or mixed FC levels (Stevens et al., 2010; Eryilmaz et al., 2011). Our study shows that both increases and decreases in FC after the cortical activation occurred and were quantitatively proportional to the level (and sign) of co-modulation. Therefore, cortical networks that showed antagonistic relationship during training (e.g., the right IPL and left dACC) showed a corresponding reduction in their resting-state FC after training. However, it is important to point out that our present study was focused on the dACC network, so generalization to other cortical networks necessitates additional verification.

Interestingly, our study shows that the cortical restructuring was significantly accentuated when long versus short delays were compared (Fig. 3B,C). This unexpected finding raises the intriguing possibility that the enhancement reflects overnight consolidation effects (Karni et al., 1994; Laureys et al., 2001; Lamprecht and LeDoux, 2004). However, it is also possible that exhaustion or fatigue processes immediately after the task reduced the full manifestation of the FC-restructuring effect.

Although we have used a NF procedure to achieve effective brain activation, there is no reason to assume that NF per se played a critical role in the effect. Furthermore, our study was not aimed to demonstrate an improved dACC control or learning effects after the NF. The significant link between the extent of coactivation and the focusing effect (Fig. $5 \mathrm{~A}$ ) suggests that the critical parameter in restructuring was the task-coactivation level rather than the NF procedure itself. However, it is possible that the intensity of the NF activations (Christopher deCharms, 2008) and the volition-related NF strategy (Preminger et al., 2011) allowed us to generate long-term changes even after 30 min of task activation.

As mentioned previously, the subjects were all research students and this might have contributed to their success in controlling the dACC by possibly having enhanced motivation to succeed in such a NF procedure. Future studies can include populations of subjects who are not research students to further generalize the effect.

It could be argued that our day-after effect might be the result of subjects' reenactment of the NF strategy during the rest period 
on the following day. Several types of analyses were conducted to rule out this interpretation. First, subjects were interviewed immediately after each of the NF and rest conditions and reported about their thought content. Detailed, blind analysis of these reports showed a significant difference between the NF and resting conditions, but no difference between any of the resting conditions themselves and no correlation between the amplitude of NF activation or the sharpening effect and the category of thought content during rest. Second, analyzing separately selected groups of subjects whose reported thought content during the resting scan appeared to greatly differ from the NF strategy (e.g., repetitive inner speech) failed to reveal a significantly lower focusing effect (see Results and Fig. 5A).

Finally, using BOLD fluctuation amplitude (power) as a "signature" of focused cognitive processes, we show that during the NF, fluctuation power was significantly different from both the pre- and day-after NF resting states. However, no significant difference was found between the pre and day-after resting-state fluctuations themselves. These results argue against the possibility that the focusing effect was due to subjects' reenactment of the NF thought processes during the resting-state periods.

Another trivial explanation for the focusing effect may be subjects' fatigue or other general time-related effects. Therefore, it could be argued that the focused FC was not a result of the NF procedure proper, but was merely due to the repetition of the fMRI scanning. Three findings argue against a "fatigue" effect. First, although we have not included a "passive" control group that did not perform the NF task, our results show that the amount of focusing effect was specifically linked to the amplitude of NF activation and not merely to the number of fMRI scans. Furthermore, the effect was selective to the NF target ROI, because repeating the same analysis on a sham ROI located near the NF ROI but not activated failed to reveal the sharpening effect (Fig. 6). Furthermore, the lack of sharpening effect in the sham ROI could not be attributed to faster habituation dynamics, because no significant focusing was found immediately after the NF period (see Results). Second, the effect was complex in nature, in fact showing both increases and decreases in resting-state FC depending on the level of coactivation during the NF session (Fig. $5 A$ ). Third, we found, in a largely data-driven manner (although it should be noted that our analysis is novel and additional research will be needed to fully establish its potential), that the focusing effect coincided with the specific NF-activated region (Fig. $7 B$ ). The anatomical specificity and activity dependence of this effect strongly argue against a generalized fatigue produced by the repeated fMRI scanning as the underlying cause of the focusing effect.

Our present results offer a unified and plausible explanation to the remarkably rich and consistent pattern of coordinated activity observed during rest. Therefore, our findings are compatible with the hypothesis that the patterning of resting-state activity is the result of a generalized Hebbian-like sharpening principle that is initiated by prior network activations. In other words, the resting-state patterns can be viewed as slowly changing traces that have been imposed, at least partially, upon the cortical architecture by the accumulated impact of prior cortical activity. How much of the resting-state connectivity pattern may be attributed to such prior Hebbian sharpening is still not clear. The actual role played by such restructuring, whether it simply reflects strengthening and weakening of FC (Amedi et al., 2005) or serve some other function (Esser et al., 2006; Schmidt et al., 2006; Tononi and Cirelli, 2006), remains to be investigated.
We demonstrate here that a focus of brain activation that occurred $24 \mathrm{~h}$ before the scan could be revealed in a data-driven and hypothesis-free analysis (Fig. $7 B$ ). Of particular relevance is the observation that this effect was extremely robust and thus could be detected in every subject tested individually. Given that the resting-state scan lasted merely 6 min, this result opens up the exciting possibility that the resting-state patterns may constitute a powerful brain-wide and personalized "window" into the personal history of brain activations in individual subjects. Such information could be important for an objective yet personalized diagnosis of cortical and cognitive pathologies and perhaps even the unique habits and traits in healthy individuals.

\section{References}

Albert NB, Robertson EM, Miall RC (2009) The resting human brain and motor learning. Curr Biol 19:1023-1027. CrossRef Medline

Amedi A, Merabet LB, Bermpohl F, Pascual-Leone A (2005) The occipital cortex in the blind-lessons about plasticity and vision. Curr Dir Psychol Sci 14:306-311. CrossRef

Artola A, Bröcher S, Singer W (1990) Different voltage-dependent thresholds for inducing long-term depression and long-term potentiation in slices of rat visual cortex. Nature 347:69-72. CrossRef Medline

Benjamini Y, Hochberg Y (1995) Controlling the false discovery rate: a practical and powerful approach to multiple testing. J Royal Stat Soc Series B (Methodological) 289-300.

Biswall B (2010) 12 Resting-state brain connectivity. Foundational Issues in Human Brain Mapping 135.

Buckner RL, Carroll DC (2007) Self-projection and the brain. Trends Cogn Sci 11:49-57. CrossRef Medline

deCharms RC (2008) Applications of real-time fMRI. Nat Rev Neurosci 9:720-729. CrossRef Medline

deCharms RC, Maeda F, Glover GH, Ludlow D, Pauly JM, Soneji D, Gabrieli JD, Mackey SC (2005) Control over brain activation and pain learned by using real-time functional MRI. Proc Natl Acad Sci US A 102:18626-18631. CrossRef Medline

Eryilmaz H, Van De Ville D, Schwartz S, Vuilleumier P (2011) Impact of transient emotions on functional connectivity during subsequent resting state: A wavelet correlation approach. Neuroimage 54:2481-2491. CrossRef Medline

Esser SK, Huber R, Massimini M, Peterson MJ, Ferrarelli F, Tononi G (2006) A direct demonstration of cortical LTP in humans: a combined TMS/EEG study. Brain Res Bull 69:86-94. CrossRef Medline

Fox MD, Snyder AZ, Vincent JL, Corbetta M, Van Essen DC, Raichle ME (2005) The human brain is intrinsically organized into dynamic, anticorrelated functional networks. Proc Natl Acad Sci U S A 102:9673-9678. CrossRef Medline

Friston KJ, Holmes AP, Price CJ, Büchel C, Worsley KJ (1999) Multisubject fMRI studies and conjunction analyses. Neuroimage 10:385-396. CrossRef Medline

Golland Y, Golland P, Bentin S, Malach R (2008) Data-driven clustering reveals a fundamental subdivision of the human cortex into two global systems. Neuropsychologia 46:540-553. CrossRef Medline

Greicius MD, Krasnow B, Reiss AL, Menon V (2003) Functional connectivity in the resting brain: a network analysis of the default mode hypothesis. Proc Natl Acad Sci U S A 100:253-258. CrossRef Medline

Hebb DO (1949) The organization of behavior: a neuropsychological theory. New York: Psychology Press.

Holroyd CB, Coles MG (2008) Dorsal anterior cingulate cortex integrates reinforcement history to guide voluntary behavior. Cortex 44:548-559. CrossRef Medline

Johnston SJ, Boehm SG, Healy D, Goebel R, Linden DE (2010) Neurofeedback: a promising tool for the self-regulation of emotion networks. Neuroimage 49:1066-1072. CrossRef Medline

Karni A, Tanne D, Rubenstein BS, Askenasy JJ, Sagi D (1994) Dependence on REM sleep of overnight improvement of a perceptual skill. Science 265:679-682. CrossRef Medline

Lamprecht R, LeDoux J (2004) Structural plasticity and memory. Nat Rev Neurosci 5:45-54. CrossRef Medline

Laureys S, Peigneux P, Phillips C, Fuchs S, Degueldre C, Aerts J, Del Fiore G, Petiau C, Luxen A, van der Linden M, Cleeremans A, Smith C, Maquet P (2001) Experience-dependent changes in cerebral functional connectiv- 
ity during human rapid eye movement sleep. Neuroscience 105:521-525. CrossRef Medline

Levy R, Dubois B (2006) Apathy and the functional anatomy of the prefrontal cortex-basal ganglia circuits. Cereb Cortex 16:916-928. CrossRef Medline

Lewis CM, Baldassarre A, Committeri G, Romani GL, Corbetta M (2009) Learning sculpts the spontaneous activity of the resting human brain. Proc Natl Acad Sci U S A 106:17558-17563. CrossRef Medline

Markram H, Lübke J, Frotscher M, Sakmann B (1997) Regulation of synaptic efficacy by coincidence of postsynaptic APs and EPSPs. Science 275: 213-215. CrossRef Medline

Mennes M, Kelly C, Zuo XN, Di Martino A, Biswal BB, Castellanos FX, Milham MP (2010) Inter-individual differences in resting-state functional connectivity predict task-induced BOLD activity. Neuroimage 50: 1690-1701. CrossRef Medline

Mostofsky SH, Simmonds DJ (2008) Response inhibition and response selection: Two sides of the same coin. J Cogn Neurosci 20:751-761. CrossRef Medline

Nir Y, Hasson U, Levy I, Yeshurun Y, Malach R (2006) Widespread functional connectivity and fMRI fluctuations in human visual cortex in the absence of visual stimulation. Neuroimage 30:1313-1324. CrossRef Medline

Nir Y, Mukamel R, Dinstein I, Privman E, Harel M, Fisch L, Gelbard-Sagiv H, Kipervasser S, Andelman F, Neufeld MY, Kramer U, Arieli A, Fried I, Malach R (2008) Interhemispheric correlations of slow spontaneous neuronal fluctuations revealed in human sensory cortex. Nat Neurosci 11:1100-1108. CrossRef Medline

Power JD, Fair DA, Schlaggar BL, Petersen SE (2010) The development of human functional brain networks. Neuron 67:735-748. CrossRef Medline

Preminger S, Harmelech T, Malach R (2011) Stimulus-free thoughts induce differential activation in the human default network. Neuroimage 54: 1692-1702. CrossRef Medline

Preminger S, Harmelech T, et al. (2008) Functional magnetic resonance imaging study of self-initiated motivated behavior in the human cerebral cortex. Soc Neurosci Abstr. 34:682.2.

Riedl V, Valet M, Wöller A, Sorg C, Vogel D, Sprenger T, Boecker H, Wohlschläger AM, Tölle TR (2011) Repeated pain induces adaptations of intrinsic brain activity to reflect past and predict future pain. Neuroimage 57:206-213. CrossRef Medline

Rushworth MF, Walton ME, Kennerley SW, Bannerman DM (2004) Action sets and decisions in the medial frontal cortex. Trends Cogn Sci 8:410417. CrossRef Medline

Salomon R, Bleich-Cohen M, Hahamy-Dubossarsky A, Dinstien I, Weizman R, Poyurovsky M, Kupchik M, Kotler M, Hendler T, Malach R (2011)
Global functional connectivity deficits in schizophrenia depend on behavioral state. J Neurosci 31:12972-12981. CrossRef Medline

Scharnowski F, Hutton C, Josephs O, Weiskopf N, Rees G (2012) Improving visual perception through neurofeedback. J Neurosci 32:17830-17841. CrossRef Medline

Schmidt C, Peigneux P, Muto V, Schenkel M, Knoblauch V, Münch M, de Quervain DJ, Wirz-Justice A, Cajochen C (2006) Encoding difficulty promotes postlearning changes in sleep spindle activity during napping. J Neurosci 26:8976-8982. CrossRef Medline

Shirer W, Ryali S, Rykhlevskaia E, Menon V, Greicius M (2011) Decoding subject-driven cognitive states with whole-brain connectivity patterns. Cereb Cortex.

Stevens WD, Buckner RL, Schacter DL (2010) Correlated low-frequency BOLD fluctuations in the resting human brain are modulated by recent experience in category-preferential visual regions. Cereb Cortex 20:19972006. CrossRef Medline

Talairach J, Tournoux P (1988) Co-planar stereotaxic atlas of the human brain: 3-dimensional proportional system: an approach to cerebral imaging (Thieme).

Tambini A, Ketz N, Davachi L (2010) Enhanced brain correlations during rest are related to memory for recent experiences. Neuron 65:280-290. CrossRef Medline

Taubert M, Lohmann G, Margulies DS, Villringer A, Ragert P (2011) Longterm effects of motor training on resting-state networks and underlying brain structure. Neuroimage 57:1492-1498. CrossRef Medline

Tononi G, Cirelli C (2006) Sleep function and synaptic homeostasis. Sleep Med Rev 10:49-62. CrossRef Medline

Urner M, Schwarzkopf DS, Friston K, Rees G (2013) Early visual learning induces long-lasting connectivity changes during rest in the human brain. Neuroimage.

Van Dijk KR, Hedden T, Venkataraman A, Evans KC, Lazar SW, Buckner RL (2010) Intrinsic functional connectivity as a tool for human connectomics: theory, properties, and optimization. J Neurophysiol 103:297-321. CrossRef Medline

Vincent JL, Snyder AZ, Fox MD, Shannon BJ, Andrews JR, Raichle ME, Buckner RL (2006) Coherent spontaneous activity identifies a hippocampal-parietal memory network. J Neurophysiol 96:3517-3531. CrossRef Medline

Weiskopf N, Scharnowski F, Veit R, Goebel R, Birbaumer N, Mathiak K (2004) Self-regulation of local brain activity using real-time functional magnetic resonance imaging (fMRI). J Physiol Paris 98:357-373. CrossRef Medline

Zhang G, Zhang H, Li X, Zhao X, Yao L, Long Z (2012) Functional alteration of the DMN by learned regulation of the PCC using real-time fMRI. IEEE Trans Neural Syst Rehabil Eng. Advance online publication. Retrieved October 9, 2012. CrossRef Medline 